\title{
Experiencia de Implantación de Estrategias de Autoevaluación y Coevaluación en el Grado de Ingeniería Telemática
}

\author{
Jaume Ramis Bibiloni, M. Magdalena Payeras Capellà, Loren Carrasco Martorell \\ Departament de Ciències Matemàtiques i Informàtica \\ Universitat de les Illes Balears \\ Carretera de Valldemossa, Km. 7,507122 Palma \\ jaume.ramis, mpayeras, loren.carrasco@uib.es
}

\begin{abstract}
Resumen-En este artículo se presenta una propuesta metodológica aplicada a la asignatura Planificación de redes del tercer curso del Grado de Ingeniería Telemática de la Universidad de las Islas Baleares. Se fundamenta en la utilización de técnicas de autoevaluación y coevaluación (evaluación entre iguales) con el objetivo de mantener a los alumnos continuamente informados sobre su proceso de aprendizaje, estimulando la cooperación entre ellos asi como su interacción con el profesor. Esta metodología consigue además mejorar su capacidad para formular críticas constructivas respecto del trabajo propio y del de los compañeros. Los resultados obtenidos ponen de manifiesto la creciente calidad de la tarea de evaluación realizada por los alumnos y evidencian la buena aceptación de esta propuesta metodológica entre ellos.
\end{abstract}

Palabras Clave-Evaluación continuada, Autoevaluación, Coevaluación, Cooperación, Rúbrica

\section{INTRODUCCIÓN}

La evaluación continuada es uno de los pilares en los que se sustenta una docencia de calidad. Se debe diferenciar entre información continuada y calificación continuada [1]. La primera es imprescindible para una docencia de calidad. En cambio, la segunda no siempre es recomendable, ya que calificar al alumno antes de la última fase del curso, cuando todavía no ha asimilado los contenidos de la asignatura, puede sesgar su calificación final. Como herramientas para lograr informar de forma continua y con prontitud a los alumnos podemos utilizar la autoevaluación y la evaluación entre iguales o coevaluación. Para lograr que esta colaboración de los alumnos en la evaluación continuada goce de buena salud es imprescindible instruirlos en el procedimiento a seguir para dicha tarea. La utilización de rúbricas constituye una buena estrategia para facilitar el proceso de evaluación. La rúbrica es un documento que delimita lo que es evaluable y lo concreta en niveles de eficiencia.

Este artículo recoge las experiencias y los resultados obtenidos en la aplicación de estrategias de autoevaluación y coevaluación en la asignatura 'Planificación de redes' del tercer curso del Grado en Ingeniería Telemática. La implantación ha sido gradual, incorporando en primer lugar la autoevaluación y, posteriormente, la coevaluación a título ilustrativo, para finalmente incorporarla como un elemento más en el proceso de evaluación. Ello responde a la utilización de diversos métodos de evaluación que pretenden no únicamente calificar al alumno sino proporcionarle herramientas para la mejora en el desarrollo de competencias.

Este proceso ha permitido, por una parte, detectar un cambio en la actitud y en el comportamiento del alumnado frente al trabajo evaluado $\mathrm{y}$, por otra, observar como las notas de la coevaluación se han ido aproximando, paulatinamente, a las notas del profesor, evidenciando que los alumnos han ido aprendiendo a utilizar las rúbricas y por tanto ha mejorado su capacidad de detección de los errores propios y ajenos. De esta manera se ha fomentado el hábito de reflexión sobre el trabajo realizado, elemento fundamental para el aprendizaje autónomo. Así mismo, la evaluación entre iguales fomenta la capacidad de emitir críticas constructivas y juzgar el trabajo de los compañeros, habilidad fundamental para el ejercicio profesional.

A continuación se describirán los métodos de evaluación utilizados antes de la adopción de la propuesta detallada en el presente trabajo. Seguidamente se explicará en qué consiste nuestra propuesta metodológica; en concreto, se presentará la rúbrica de evaluación adoptada y el procedimiento que se ha seguido para la familiarización de los alumnos con su utilización. Se mostrarán después los resultados obtenidos, que serán analizados con el objetivo de valorar la efectividad de la metodología planteada. Para finalizar se concluirá el artículo con una reflexión sobre su aplicación práctica y las posibilidades de mejora. 


\section{Metodología de EVAluación PREVIA}

Los contenidos de la asignatura Planificación de Redes del tercer curso del Grado en Ingeniería Telemática consisten básicamente en el modelado formal de protocolos mediante la utilización de cadenas de Markov. La metodología didáctica utilizada se basa fundamentalmente en la realización de explicaciones teóricas por parte del profesor y en la resolución de problemas y realización de prácticas por parte de los alumnos con el objetivo de consolidar los conceptos y conocimientos introducidos por el profesor. Los alumnos trabajan en grupos reducidos, generalmente de dos miembros, y deben entregar sus actividades unos días después de finalizar cada tema, según marca el calendario indicado por el profesor, a través de Campus Extens (plataforma Moodle). La resolución de ejercicios y prácticas se lleva a cabo mediante la realización de pequeños programas utilizando el Matlab [2]. Además se realizan dos controles a lo largo del cuatrimestre, siendo necesario superar ambas pruebas (nota superior a cinco) para aprobar la asignatura.

Uno de los siete principios de la docencia de calidad [3] consiste en proporcionar retroalimentación a tiempo. Es por ello que resulta imprescindible disponer de las herramientas necesarias para mantener al alumno informado sobre su progreso a lo largo de todo el curso, sin que tenga que esperar a los exámenes para ello. Así pues, el alumno debe disponer de esta retroalimentación de forma rápida para poder emprender las acciones que considere oportunas.

Con este objetivo, en el primer año de impartición de la asignatura Planificación de Redes, ésta se diseñó de manera que tras cada entrega, consistente en el conjunto de actividades correspondientes a un tema, el profesor llevaba a cabo su corrección y posteriormente informaba a los alumnos de la calificación obtenida por su trabajo. Dada la naturaleza de los trabajos, este esquema resultó del todo ineficiente e improducivo: por una parte implicó una elevada carga de trabajo para el profesor, dada la gran cantidad de ejercicios a resolver a pesar del no muy elevado número de alumnos $\mathrm{y}$, por otro lado, tan sólo consitía en una calificación continuada y estaba lejos de ser una información continuada.

A partir de la segunda edición del curso, se decidió mantener la realización de las mismas entregas de actividades por parte del alumnado, pero se sustituyó la corrección de todos los ejercicios por la corrección de una muestra de ejercicios seleccionados por el profesor. Se trataba de una muestra suficientemente respresentativa de la tipología de actividades de las entregas realizadas por los alumnos. Entonces el profesor llevaba a cabo la corrección de esta selección de ejercicios y asignaba la calificación correspondiente. Con esta estrategia se redujo considerablemente la carga de trabajo del profesor sin que ello respresentara una reducción significativa de la retroalimentación que recibían los alumnos. Pero los alumnos seguían sin disponer de la información continuada sobre su progreso.

Este esquema se mantuvo hasta el curso pasado, cuando se decidió modificar la metodología didáctica con el objetivo de proporcionar una retroalimentación de calidad a los alumnos, apostando por una estrategia que estimulase además la cooperación entre ellos y que favoreciese el contacto entre alumnos y profesor, elementos que también se encuentran entre los siete principios de la docencia de calidad [3]. En la siguiente sección se detalla en qué consite esta propuesta.

\section{Metodología PROPUESTA}

La metodología didáctica propuesta incorpora la autoevaluación y la coevaluación (o evaluación entre compañeros) como herramientas que facilitan la retroalimentación a tiempo y de forma continuada a los alumnos sobre el progreso de su trabajo a lo largo de todo el curso. Para ello es fundamental la utilización de una rúbrica para la evaluación de los programas realizados por los alumnos utilizando la herramienta Matlab [2]. Resulta imprescindible que el evaluador, ya sea el propio autor del trabajo, un compañero de clase o bien el profesor, haga suya la rúbrica. Sólo así se logrará el éxito de la presente propuesta.

\section{A. Rúbrica para la evaluación}

La rúbrica utilizada se muestra en la Tabla I. Se halla estructurada en tres columnas, la primera de las cuales especifica cada criterio de evaluación considerado, mientras que la segunda corresponde a una descripción detallada de los aspectos que se deben considerar para el criterio en cuestión y, finalmente, la tercera especifica cómo asignar la puntuación.

De acuerdo con los criterios considerados, se valora la estructura y claridad del código así como su corrección. En cuanto a los resultados se valora tanto la claridad en la presentación de los resultados como su corrección, así como la interpretación de los mismos.

La rúbrica no tan sólo es un herramienta para la evaluación sino también para el aprendizaje, puesto que detalla las características que debe satisfacer un trabajo para ser evaluado de forma positiva. Su validez depende de la objetividad en su aplicación, permitiendo a distintos evaluadores asignar puntuaciones muy similares a un mismo trabajo. La existencia de diferencias significativas en las evaluaciones puede denotar la falta de objetividad en la rúbrica y/o el poco dominio del evaluador en su utilización.

\section{B. Procedimiento}

Tanto la planificación de la asignatura como su evaluación se diseñaron dando máxima prioridad a la familiarización del alumnado con la utilización de la rúbrica de evaluación. Así pues, la asignatura se divide en dos grandes bloques: el primero de conceptos básicos y el segundo de modelado formal de protocolos. Como ya se ha comentado en la sección II, a lo largo de todo el curso los alumnos trabajan en grupos de dos alumnos y van realizando las entregas de las actividades de cada tema. Además, tras finalizar cada bloque, los alumnos 
Tabla I

RÚBRICA ORIGINAL UTILIZADA EN LA ASIGNATURA "PLANIFICACIÓN DE REDES"

\begin{tabular}{|c|c|c|}
\hline Criterio & Descripción & Puntuación \\
\hline $\begin{array}{l}\text { Estructura y claridad del código } \\
\text { ( } 2 \text { puntos) }\end{array}$ & $\begin{array}{l}\text { El código esta organizado y estructurado correctamente } \\
\text { El código está identado correctamente. } \\
\text { Las instrucciones incluyen descripciones explicativas y aclaradoras. }\end{array}$ & $\begin{array}{l}2 \text { puntos si se cumplen todos los criterios } \\
1 \text { punto si falta un criterio por cumplir. } \\
0 \text { puntos si faltan dos o más criterios por cumplir. }\end{array}$ \\
\hline Corrección del código (3 puntos) & No hay errores en el código & $\begin{array}{l}3 \text { puntos si el número de errores es } 0 \\
2 \text { puntos si el número de errores es } 1 \\
1 \text { puntos si el número de errores es } 2 \\
0 \text { puntos si el número de errores es } 3 \text { o más }\end{array}$ \\
\hline $\begin{array}{l}\text { Resultado de la ejecución del código } \\
\text { ( } 2 \text { puntos) }\end{array}$ & $\begin{array}{l}\text { No hay duda de cual es el resultado obtenido con la ejecución. } \\
\text { Todos los gráficos incluyen la descripción de los ejes y la leyenda } \\
\text { En caso de que se requieran diversos gráficos, utilizar subplots }\end{array}$ & $\begin{array}{l}2 \text { puntos si se cumplen todos los criterios } \\
1 \text { punto si falta un criterio por cumplir } \\
0 \text { puntos si faltan dos o más criterios por cumplir }\end{array}$ \\
\hline $\begin{array}{l}\text { Corrección de los resultados } \\
(2 \text { puntos })\end{array}$ & los resultados (numéricos y gráficos) son correctos. & $\begin{array}{l}2 \text { puntos si se cumple en el } 100 \% \text { de los resultados. } \\
1 \text { punto si se cumple en más del } 75 \% \text {. } \\
0 \text { puntos si se cumple en menos del } 75 \% \text {. }\end{array}$ \\
\hline $\begin{array}{l}\text { Comentarios de los resultados } \\
\text { (1 punto) }\end{array}$ & Los resultados están completamente y correctamente comentados & $\begin{array}{l}2 \text { puntos si se cumple en el } 100 \% \text { de los resultados. } \\
1 \text { punto si se cumple en más del } 75 \% \text {. } \\
0 \text { puntos si se cumple en menos del } 75 \% \text {. }\end{array}$ \\
\hline
\end{tabular}

realizan, de forma individual, sendas pruebas de control. A continuación se detalla la organización del procedimiento.

1) Aprendizaje del uso de la rúbrica y autoevaluación de las entregas de actividades: Con el objetivo de familiarizar a los alumnos con la rúbrica, a lo largo de la primera parte del curso, correspondiente al bloque de conceptos básicos, tras cada entrega de actividades se destina la siguiente hora de clase a su corrección. El profesor selecciona un alumno para cada ejercicio, quien explica su resolución al grupo con la ayuda del proyector (cada alumno realizará esta intervención en público como mínimo dos veces a lo largo del curso). Seguidamente el profesor realiza los comentarios oportunos y los compañeros pueden consultarle cualquier aspecto que sea de su interés. A continuación se procede a la calificación del ejercicio utilizando la rúbrica de evaluación: alumnos y profesor consensuan las puntuaciones de cada apartado, aclarando cualquier duda sobre el procedimiento. Las calificaciones así obtenidas constituirán la nota de seguimiento del curso, con un peso del $10 \%$ sobre la nota final. Para acabar el proceso, cada alumno se autoevalúa su trabajo. Se establece como requisito indispensable para la validez de las evaluaciones que se justifiquen convenientemente las calificaciones asignadas en cada apartado de la rúbrica.

Tras finalizar la sesión de revisión de la entrega de actividades en cuestión, los alumnos, de forma individual, suben a 'Campus Extens' el resultado de la autoevaluación de su entrega. Con el objetivo de alentar a los alumnos a que realizen esta tarea de autoevaluación, un $10 \%$ de la nota final de la asignatura se corresponde a la nota por las entregas realizadas. Ésta se obtiene en función del número de entregas de actividades con sus respectivas autoevaluaciones que hayan llevado a cabo los alumnos y no de las calificaciones obtenidas en ellas. Con ello se pretende potenciar la aplicación objetiva de los criterios de calificación especificados en la rúbrica. Además, los alumnos son informados de que estas autoevaluaciones son revisadas por el profesor, quien, si lo cree necesario, puede proponer a aquellos alumnos que considere recomendable, la realización de problemas complementarios y tutorías para mejorar sus resultados.

El número total de entregas a realizar a lo largo del curso es 10. La distribución de calificaciones es la sigu- iente:

- El $100 \%$ de entregas + autoevaluación: $10 \%$

- Entre $100 \%$ y el $90 \%$ de entregas + autoevaluación: $7.5 \%$

- Entre $90 \%$ y el $80 \%$ de entregas + autoevaluación: $5 \%$

- Menos del 80\% de entregas + autoevaluación: 0\%

2) Prueba ilustrativa de autoevaluación y coevaluación del primer control: Tras este proceso, que comprende unas siete semanas, se realiza la primera prueba de control, cuyo peso sobre la nota final es del $40 \%$. Los alumnos suben sus resoluciones a 'Campus Extens', donde se halla la tarea Controll, que consiste en un taller de la plataforma Moodle [4]. Esta herramienta permite configurar actividades para la corrección entre iguales, obteniéndose una calificación de la actividad realizada por el alumno y una calificación de la tarea de evaluación de sus compañeros. Además permite incorporar cualquier tipo de rúbrica para el proceso de evaluación. La calificación del taller está configurada para que la nota del control se obtenga de la siguiente manera:

- la calificación de la actividad representa el $80 \%$, correspondiente a:

- calificación asignada por el profesor: $75 \%$

- calificación media asignada por los compañeros: $25 \%$

- la calificación media de la tarea de evaluación de sus compañeros: $20 \%$

Con el objetivo de primar la evaluación realizada por el profesor, el peso de su calificación de la actividad es tres veces mayor que el peso correspondiente a la calificación de la actividad por parte de los compañeros. Así, la calificación asignada por el profesor corresponde al $60 \%$ de la nota del control, la calificación recibida por parte de sus compañeros representa el $20 \%$ y su tarea de evaluación de los compañeros corresponde al $20 \%$ restante. Como se observa, estos dos últimos porcentajes son iguales, lo cual significa que evaluar correctamente a sus compañeros representa la misma recompensa que sobrepuntuar a sus compañeros. Con ello se incita a los alumnos a corregir de la forma más objetiva posible.

La clase posterior a la realización del control se destina a su evaluación. Ésta comienza con la resolución de 


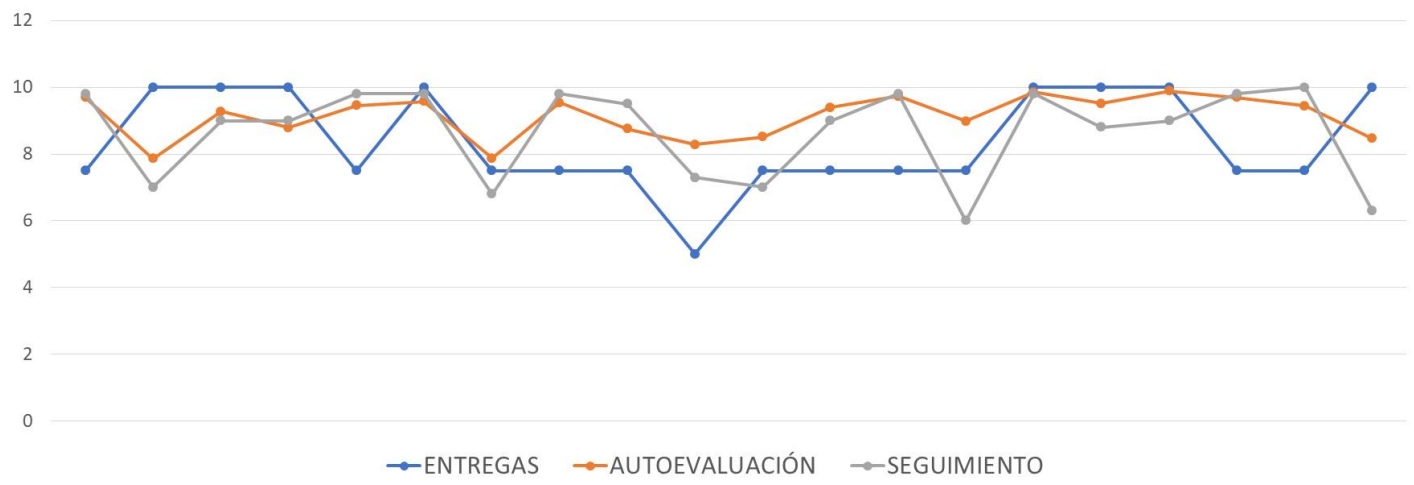

Fig. 1. Resultados del seguimiento de las entregas de actividades de los alumnos

los ejercicios del control por parte del profesor ante el grupo de clase. Seguidamente cada alumno realiza su autoevaluación y la evaluación de tres compañeros. Para ello utilizan la rúbrica incorporada en el taller de Moodle, siendo imprescindible que los alumnos justifiquen sus calificaciones mediante los comentarios oportunos.

Como primer paso en la incoroporación de la autoevaluación y la coevaluación, se decidió llevar a cabo este proceso a nivel tan sólo ilustrativo, haciendo que la calificación de esta primera prueba de control se correspondiese exclusivamente con la calificación del profesor.

3) Autoevaluación de las entregas de actividades: A lo largo de la segunda parte del curso, correspondiente al bloque de modelado formal de protocolos, los alumnos siguen realizando sus entregas de actividades y siguen llevando a cabo su resolución en clase. En este punto del curso los alumnos están altamente familiarizados con el uso de la rúbrica, de manera que durante la sesión de resolución de los ejercicios en clase, las explicaciones de los alumnos seleccionados por el profesor se centran básicamente en un comentario general del programa y de los resultados obtenidos. El profesor realiza los comentarios oportunos y, a continuación, cada alumno se autoevalúa su trabajo - no existe ya la necesidad de aplicar la rúbrica de forma conjunta y consensuada.

4) Autoevaluación y coevaluación del segundo control: La última semana se destina a la realización de la segunda prueba de control y a su evaluación, siguiendo el mismo proceso descrito para la primera prueba. Ahora ya sí que la calificación obtenida corresponde al resultado del procedimiento antes detallado.

\section{RESUltados}

El número de alumnos matriculados el curso 2016/2017 ha sido 26, de los cuales 20 alumnos han asistido a más del $80 \%$ de clases. Los 6 restantes son alumnos a tiempo parcial y no se han adherido al itinerario presencial, por lo que no han seguido la propuesta metodológica objeto del presente trabajo.

En esta sección analizaremos los resultados obtenidos, que se han organizado de la siguiente manera:
- familiarización con la rúbrica e incorporación de la autoevaluación,

- autoevaluación y coevaluación de las pruebas de control,

- resultados finales.

\section{A. Autoevaluación de las entregas de actividades}

Como ya se ha indicado en la sección III-B, la calificación obtenida por los alumnos dependerà del número de entregas de actividades y autoevaluaciones, y no de las calificaciones obtenidas en ellas. La asignación de calificaciones es muy estricta, propiciando que los alumnos lleven a cabo la mayoría de sus entregas de actividades, lo que se refleja en los resultados obtenidos, que se muestran en la Figura 1. El eje horizontal de todas las figuras se corresponde con cada uno de los 20 alumnos que han seguido la metodología propuesta en este trabajo. Exceptuando un alumno, que realizó el $85 \%$ de las entregas, el resto supera el $90 \%$ y casi la mitad llega al $100 \%$. Se representan también las calificaciones medias obtenidas en las autoevaluaciones de todas las entregas de actividades a lo largo del curso, así como la nota de seguimiento asignada por el profesor según del procedimiento detallado en la subsección III-B1. En general, se aprecian las mismas oscilaciones en estas dos últimas curvas, evidenciando la correlación existente entre ambas. La discrepancia promedio entre ellas es inferior a 0.5 puntos.

\section{B. Autoevaluación y coevaluación de las pruebas de con- trol}

En primer lugar, cabe destacar que la implementación del proceso mediante la herramienta 'taller' de la plataforma Moodle, ha facilitado la utilización de la rúbrica en la autoevaluación y coevaluación de las pruebas de control.

Debe tenerse en cuenta que la calificación de la tarea de evaluación no corresponde a la simple comparación de la calificación otorgada por el alumno coevaluador en relación a la asignada por parte del profesor. De hecho la comparación se realiza con respecto a la media ponderada de todas las calificaciones tal como se detalló en la subsección III-B2, donde se explicó que se asignan 


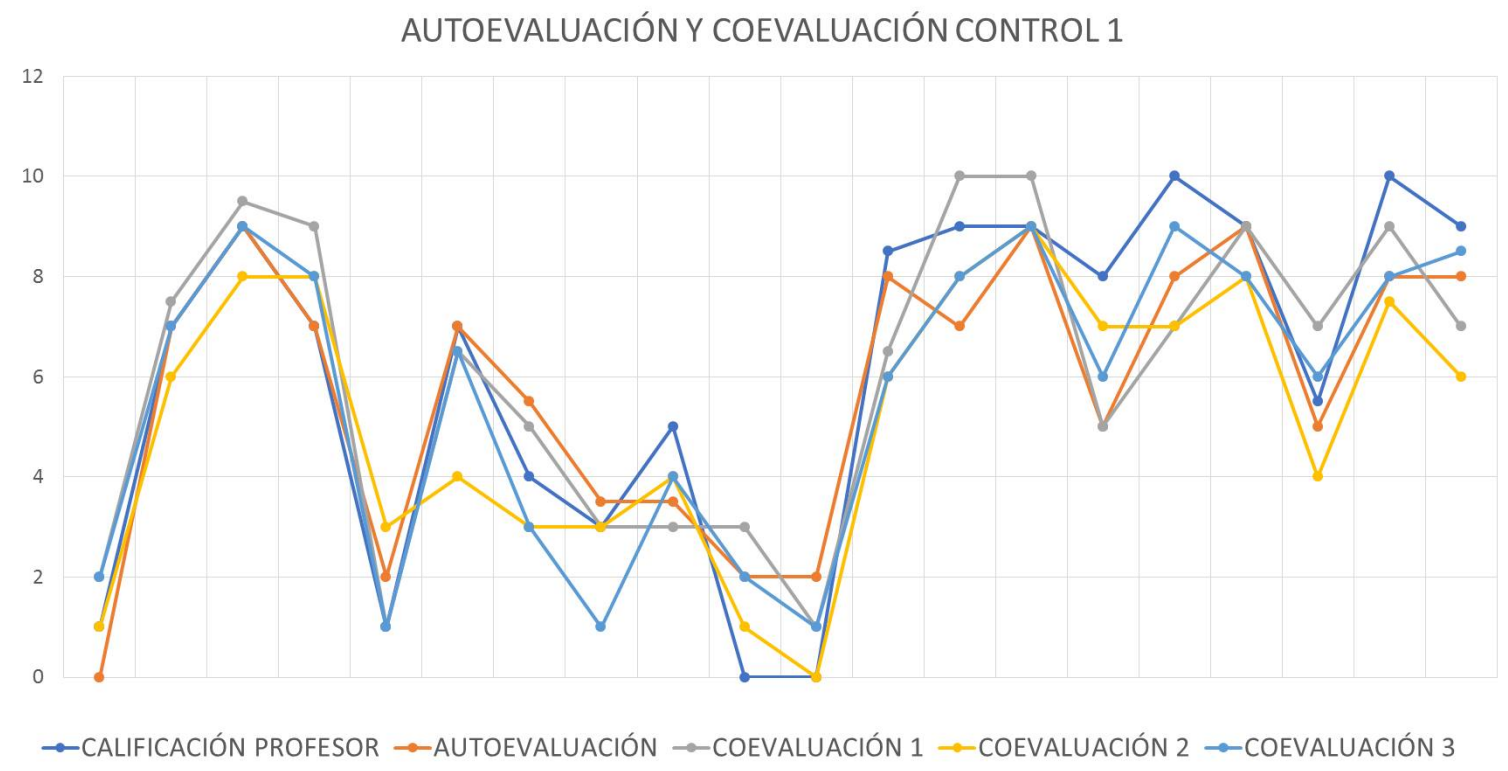

Fig. 2. Resultados de la evaluación de la primera prueba de control

pesos con el objetivo de primar la evaluación realizada por el profesor. Entonces, la tarea de evaluación se califica teniendo en cuenta las puntuaciones asignadas a cada criterio de evaluación presente en la rúbrica. Ello puede dar como resultado que la puntuación global del ejercicio según la evaluación del profesor coincida con la del alumno coevaluador, pero que sin embargo la calificación de la tarea de evaluación del alumno reciba una puntuación muy baja. Se trata por tanto de puntuar cada ítem de la rúbrica de forma correcta y no simplemente de asignar la nota de forma general al ejercicio.

En nuestro caso cada ejercicio ha sido evaluado por su autor, por el profesor y por tres compañeros. El análisis de los resultados representados en las figuras 2 y 3 muestran como, en general, convergen las calificaciones otorgadas por los cinco revisores. Puede observarse claramente que para el segundo control existe una mayor similitud entre todas ellas. Se aprecia que tan sólo existen muy pocos casos con discrepancias significativas (detectamos diferencias de hasta 3 puntos (sobre 10) para el primer control y de hasta 2 puntos en el segundo). Estos resultados ponen de manifiesto el progresivo dominio de los alumnos en la correcta utilización de la rúbrica. Cabe destacar además que el número de coevaluaciones realizadas en

\section{AUTOEVALUACIÓN Y COEVALUACIÓN CONTROL 2}

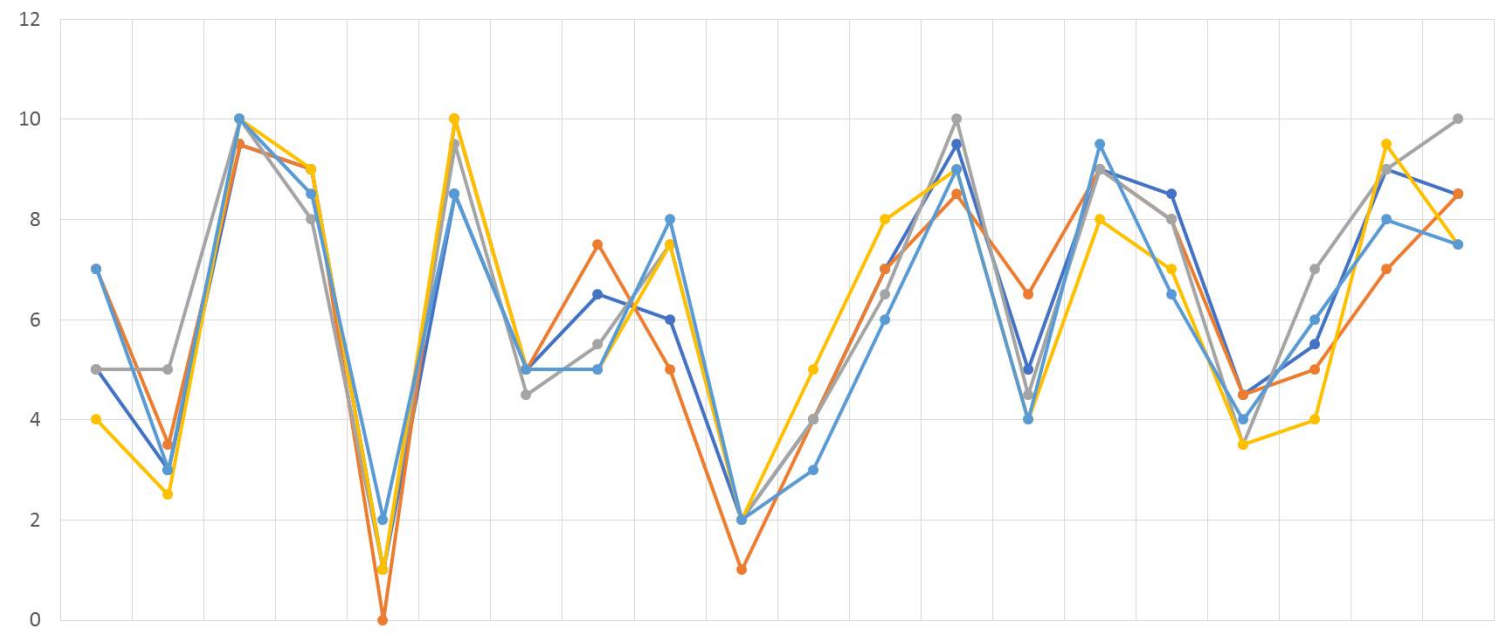

$\rightarrow$-CALIFICACIÓN PROFESOR $\rightarrow$-AUTOEVALUACIÓN $\rightarrow$-COEVALUACIÓN $1 \rightarrow$ COEVALUACIÓN $2 \rightarrow$-COEVALUACIÓN 3

Fig. 3. Resultados de la evaluación de la segunda prueba de control 
las pruebas de control debe ser suficientemente grande para garantizar la fiabilidad del proceso. Para los casos en que se producen discrepancias significativas entre las calificaciones asignadas existe la posibilidad de solicitar una segunda revisión más exhaustiva de los ejercicios al revisor en cuestión. Incluso podría descartarse dicha revisión divergente. No olvidemos que una tarea de evaluación incorrecta se traduce en una peor nota para el revisor.

Las figuras 4 y 5 representan las notas obtenidas en las dos pruebas de control, detallando la calificación de la actividad y la calificación de la tarea de evaluación. Además se incluyen las calificaciones asignadas por el profesor. En ambas figuras se aprecia un elevado grado de simililitud entre la calificación del profesor y la asignada a la actividad, básicamente coincidentes en la segunda prueba de control. En cuanto a la calificación de la tarea de evaluación, se aprecia una clara mejora en el segundo control en comparación con el primero. En el primer control el $75 \%$ de los alumnos realizaron esta tarea de forma satisfactoria (nota de la tarea de evaluación superior a 5), alcanzándose el $100 \%$ en el segundo control. Ello influye de forma positiva en la nota obtenida por los alumnos: si bien en el primer control la tarea de evaluación influyó negativamente en el $65 \%$ de los alumnos con una reducción promedio de -0.85 puntos en su nota del control, en el segundo control este porcentaje se reduce al $50 \%$ con una reducción promedio de tan solo -0.21 puntos. Los resultados ponen de manifiesto la fiabilidad del proceso, sin necesidad de descartar ninguna revisión. Ello es fruto del elevado número de revisiones de cada ejercicio.

\section{Comentario de las calificaciones del curso}

La figura 6 resume las calificaciones obtenidas por los alumnos. Recordemos que la nota final se obtiene a partir de la siguiente distribución:

- $10 \%$ nota por las entregas realizadas, de acuerdo a lo descrito en la subsección III-B1

- $10 \%$ nota de seguimiento, según se especifica en la subsección III-B1

- $40 \%$ prueba de control 1 , tal como se detalla en la subsección III-B2

- $40 \%$ prueba de control 2

Todos los alumnos han recibido una valoración positiva del número de entregas realizadas y del seguimiento. En la segunda prueba de control se aprecia una mejora en las calificaciones en el $70 \%$ de los alumnos con respecto al primer control. El número de alumnos que superan positivamente la asignatura es del $80 \%$ en la convocatoria ordinaria, resultado altamente satisfactorio.

\section{ANÁlisis DE LA EXPERIENCIA, PROPUESTAS DE MEJORA Y CONCLUSIONES}

La experiencia nos muestra que un elemento clave para el éxito de la propuesta es el diseño de una rúbrica fácilmente utilizable por parte de alumnos y profesores, que defina los criterios de evaluación de forma clara y objetiva. Además debe evitarse cualquier tipo de solape entre los criterios a evaluar. Es también fundamental distribuir los pesos proporcionalmente a la importancia que se le otorga a cada criterio. El diseño propuesto inicialmente debe ser mejorado a partir de la experiencia de su utilización. Para ello puede contarse con la colaboración de los alumnos. En el caso que nos atañe se decidió modificar la rúbrica para su utilización en la siguiente edición de la asignatura. La rúbrica mejorada se muestra en la tabla II. Se diferencia de la versión original en dos aspectos:

- se iguala el peso de todos los criterios excepto el de 'corrección del código' al que se le otorga mayor predominancia;

- se describe con mayor detalle el criterio 'corección del código' con el fin de distinguir entre errores leves $\mathrm{y}$ errores graves, especificando a título ilustrativo algunos ejemplos de cada tipología.

Además se hizo evidente que resulta imprescindible elaborar los enunciados de los ejercicios teniendo en todo momento presente que deberán ser evaluados utilizando la rúbrica. Así pues, el diseño de los ejercicios seguirá una estructura que facilitará la identificación de cada uno de los criterios detallados en la rúbrica así como su evaluación siguiendo las pautas en ella detalladas. Esto resulta especialment relevante en los ejercicios que conforman las pruebas de control.

La aplicación de la metodología propuesta en el presente trabajo ha resultado una herramienta altamente eficaz para alcanzar el objetivo que nos habíamos propuesto como primer propósito: proporcionar retroalimentación de calidad y a tiempo. La autoevaluación de cada una de las entregas de actividades a lo largo del curso ha representado un cambio muy significativo en la dinámica con respecto a las ediciones anteriores. Los alumnos perciben la autoevaluación de forma positiva dado que la rúbrica representa una guía para el aprendizaje, puesto que detalla las características que debe satisfacer su trabajo para ser evaluado de forma positiva y, al mismo tiempo, les informa continuamente sobre la calidad y la evolución de su desempeño.

Los alumnos han interiorizado la rúbrica con gran facilidad, lo que ha sido un elemento clave para el éxito de esta propuesta. Gracias a ello la calidad de las autoevaluaciones y coevaluaciones ha sido en general elevada. Los principales beneficios obtenidos son [1], [5]:

- Los alumnos hacen suyos los criterios de evaluación y mejoran cada vez más sus respuestas, fomentando el hábito de reflexión sobre el trabajo realizado, elemento fundamental para el aprendizaje autónomo.

- En cuanto a las coevaluaciones, permiten a los alumnos ver soluciones alternativas a los ejercicios, contribuyendo también a la reflexión no sólo sobre el trabajo de los demás, sino incluso sobre el propio. El hecho de ser evaluados por sus compañeros les motiva para realizar un buen trabajo. De la misma manera, evaluar a sus compañeros fomenta su capacidad de ser crítico con el trabajo de los demás.

Otro beneficio de esta propuesta metodológica ha sido que ha representado un verdadero estímulo para la coop- 
12

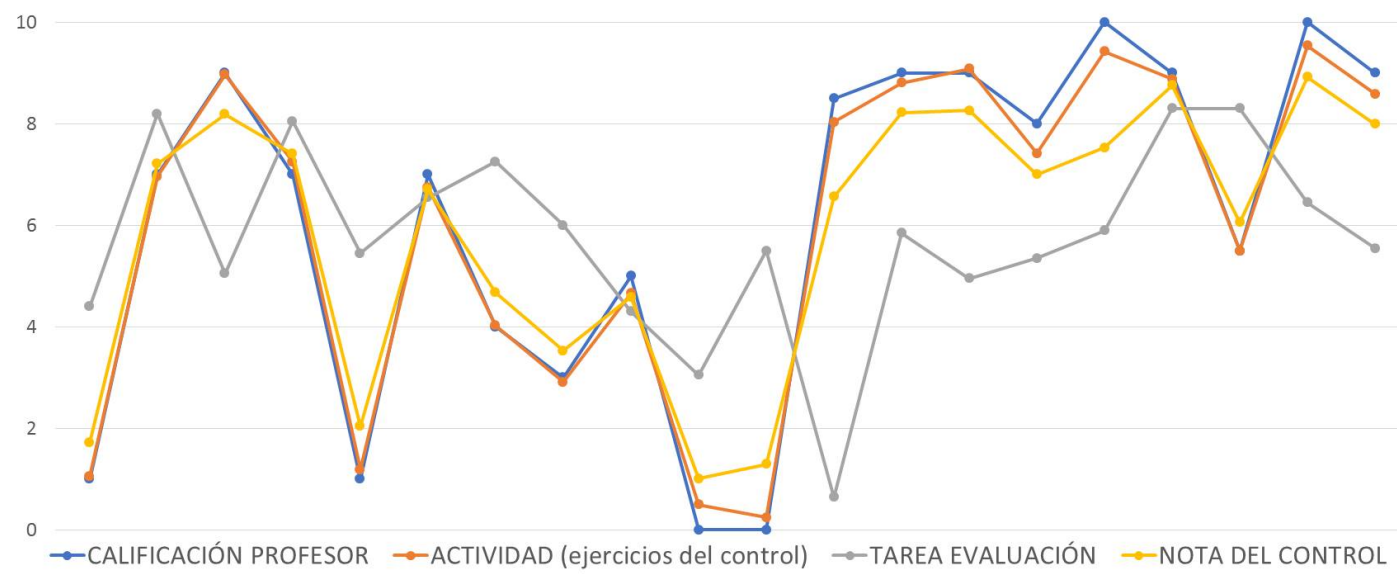

Fig. 4. Resultados de la primera prueba de control: desglose y comparativa con la calificación del profesor

Tabla II

RÚBRICA ADAPTADA UTILIZADA EN LA ASIGNATURA "PlanifiCACIÓN DE REDES"

\begin{tabular}{|c|c|c|}
\hline Criterio & Descripción & Puntuación \\
\hline Estructura y claridad del código (1,5 puntos) & $\begin{array}{l}\text { El código esta organizado y estructurado correctamente } \\
\text { El código está identado correctamente. } \\
\text { Las instrucciones incluyen descripciones explicativas y aclaradoras. }\end{array}$ & $\begin{array}{l}, 5 \text { puntos si se cumplen todos los criterios } \\
0,75 \text { puntos si falta un criterio por cumplir. } \\
0 \text { puntos si faltan dos o más criterios por cumplir. }\end{array}$ \\
\hline Corrección del código (4 puntos) & $\begin{array}{l}\text { Se distinguen dos tipos de errores. } \\
\text { Errores Graves: de concepto. } \\
\text { Errores Leves: bucles innecesarios, } \\
\text { repeticiones, pequeños errores de comando,... }\end{array}$ & $\begin{array}{l}4 \text { puntos si el número de errores es } 0 \\
\text { Cada error grave resta dos puntos. } \\
\text { Cada error leve resta un punto. }\end{array}$ \\
\hline Resultado de la ejecución del código (1,5 puntos) & $\begin{array}{l}\text { No hay duda de cual es el resultado obtenido con la ejecución. } \\
\text { Todos los gráficos incluyen la descripción de los ejes y la leyenda } \\
\text { En caso de que se requieran diversos gráficos, utilizar subplots }\end{array}$ & $\begin{array}{l}1,5 \text { puntos si se cumplen todos los criterios } \\
0,75 \text { puntos si falta un criterio por cumplir } \\
0 \text { puntos si faltan dos o más criterios por cumplir }\end{array}$ \\
\hline Corrección de los resultados (1,5 puntos) & los resultados (numéricos y gráicos) son correctos. & $\begin{array}{l}1,5 \text { puntos si se cumple en el } 100 \% \text { de los resultados. } \\
0,75 \text { puntos si se cumple en más del } 75 \% \text {. } \\
0 \text { puntos si se cumple en menos del } 75 \% \text {. }\end{array}$ \\
\hline Comentarios de los resultados (1,5 puntos) & $\begin{array}{l}\text { Los resultados (numéricos y gráficos) } \\
\text { estan completamente y correctamente comentados }\end{array}$ & $\begin{array}{l}1,5 \text { puntos si se cumple en el } 100 \% \text { de los resultados. } \\
0,75 \text { punto si se cumple en más del } 75 \% \text {. } \\
0 \text { puntos si se cumple en menos del } 75 \% \text {. }\end{array}$ \\
\hline
\end{tabular}

eración entre alumnos. El trabajo en equipo ha resultado un elemento clave en el proceso de aprendizaje de la utilización de la rúbrica de evaluación. Además ha favorecido el contacto entre alumnos y profesor, puesto que éste ha sido quien continuamente ha aclarado todo tipo de dudas en cuanto a la aplicación de la rúbrica. Las consultas al

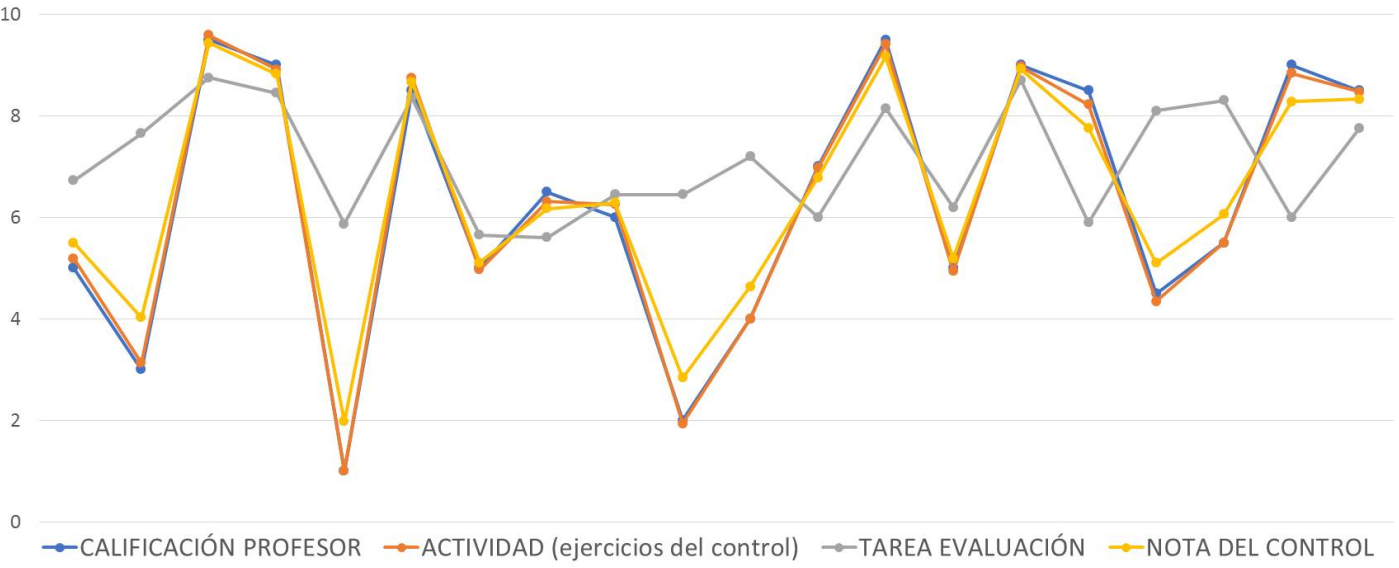

Fig. 5. Resultados de la segunda prueba de control: desglose y comparativa con la calificación del profesor 


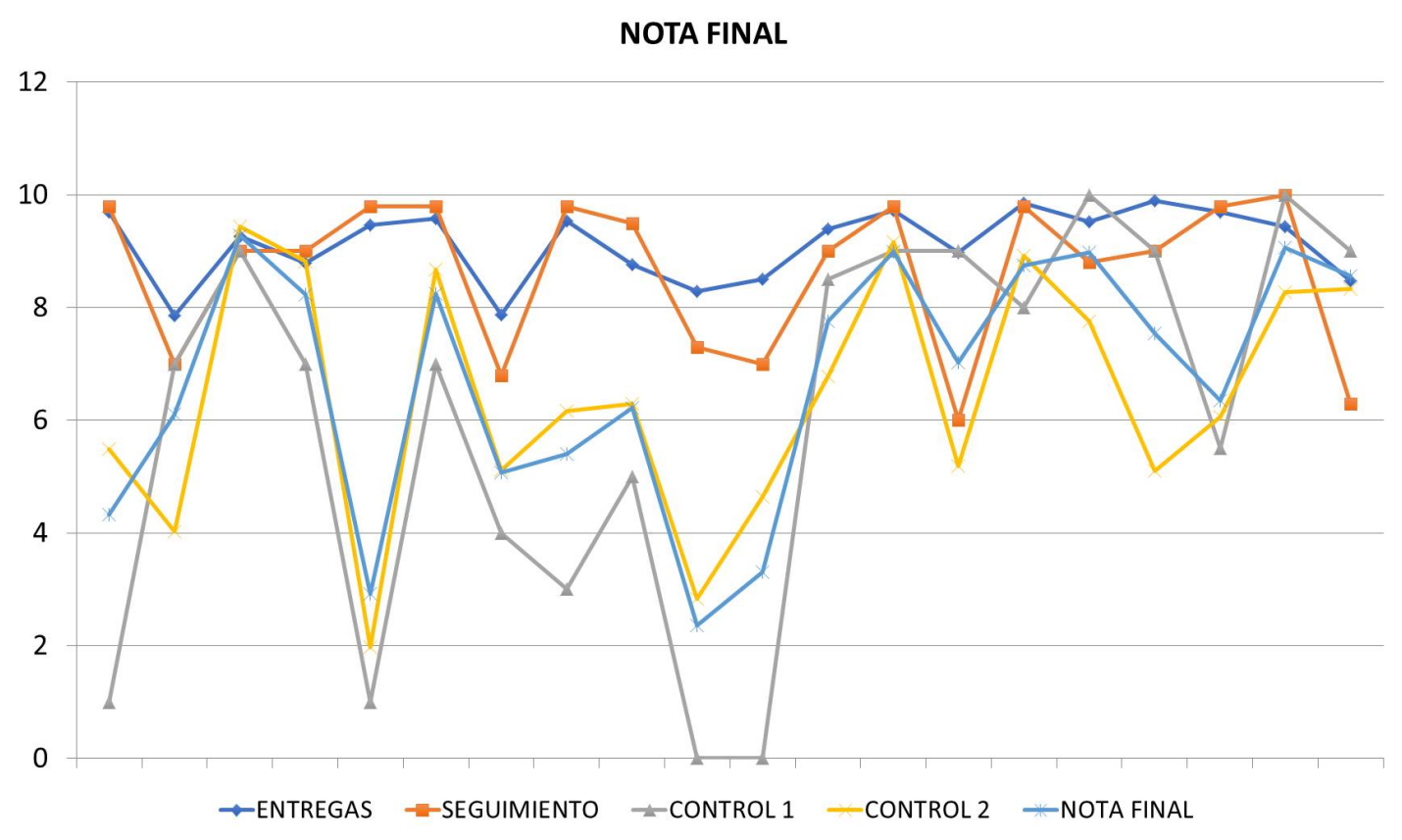

Fig. 6. Resultados de la calificación final del curso

profesor han proliferado de forma especialmente significativa durante las sesiones de autoevaluación y coevaluación de las pruebas de control, dado el interés de los alumnos en aplicar correctamente la rúbrica para obtener así una buena calificación de su tarea de evaluación. En definitiva, la participación del alumnado en el desarrollo de todo el proceso de aprendizaje, tanto autónomo como en clase, ha sido mucho más activa.

Como evidencia objetiva del grado de aceptación de esta propuesta metodológica, la tabla III muestra la evolución del resultado correspondiente al ítem 'nivel de satisfacción' del informe de opinión del alumnado de grado sobre la tarea docente del profesorado, aplicado por el Servei d'Estadística i Qualitat Universitària [6]. Puede observarse la significativa mejora en el nivel de satisfacción de los alumnos correspondiente a la última edición del curso con respecto a la anterior. Ello evidencia la buena acogida de esta propuesta metodológica entre el alumnado.

Tabla III

NiVEL DE SATISFACCIÓN DEL ALUMNADO DE LA ASIGNATURA 'PLANIFICACIÓN DE REDES'

\begin{tabular}{|l|l|l|l|l|}
\hline $\mathbf{2 0 1 2 / 2 0 1 3}$ & $\mathbf{2 0 1 3 / 2 0 1 4}$ & $\mathbf{2 0 1 4 / 2 0 1 5}$ & $\mathbf{2 0 1 5 / 2 0 1 6}$ & $\mathbf{2 0 1 6 / 2 0 1 7}$ \\
\hline 7,25 & 5,91 & 7,17 & 6,7 & 8,32 \\
\hline
\end{tabular}

Es obvio el ahorro en el trabajo de revisión de las entregas de actividades por parte del profesor y la mejora en la calidad de la retroalimentación, a tiempo y de forma continuada, que reciben los alumnos sobre el progreso de su trabajo a lo largo de todo el curso. De todas formas, ha sido necesario invertir esfuerzos por ambas partes, alumnos y profesor, para la aplicación en el aula de esta metodología didáctica. Han sido necesarias 8 horas para llevar a cabo las sesiones de revisión de las actividades en clase, lo que ha significado un incremento de las horas de trabajo autónomo de los alumnos, puesto que los ejercicios no se han reducido con respecto a las ediciones anteriores de la asignatura. Por otra parte, el profesor ha visto incrementada significativamente la carga horaria asociada a la impartición de la asignatura debido al diseño de la metodología en sí, la configuración de la rúbrica, la implementación de los talleres de Moodle para las pruebas de control y el seguimiento y control de todo el procedimiento. A pesar de ello, la buena aceptación por parte del alumnado, los satisfactorios resultados académicos obtenidos y las expectativas de seguir aplicando esta metodología a lo largo de los próximos cursos, compensan con creces el esfuerzo realizado.

Para finalizar, cabe indicar que se pueden utilizar procedimientos similares a la metodología aquí propuesta a otra tipología de trabajos, como pueden ser colecciones de problemas, informes o trabajos escritos o también presentaciones orales. En este sentido los autores están perfilando el diseño de estrategias de coevaluación para presentaciones orales de trabajos en equipo.

\section{REFERENCIAS}

[1] Miguel Valero-García, Luis M. Díaz de Cerio, Autoevaluación y coevaluación: estrategias para facilitar la evaluación continuada, Actas del Simposio Nacional de Docencia en la Informática, SINDI2005 (AENUI), pp.25-32 ISBN: 84-9732-443-9, 2005

[2] https://www.mathworks.com/products/matlab.html

[3] A. W. Chickering y Z. F. Gamson, Seven Principles for Good Practice in Undergraduate Education, March 1987, American Association of Higher Education and Accreditation (AAHEA) Bulletin, Vol. 39(7), pages 3-7

[4] https://moodle.org

[5] A.W. Bangert, Peer Assessment: A Win-Win Instructional Strategy for Both Students and Teachers, J. Cooperation \& Collaboration in College Teaching, Vol. 10, No. 2, pp. 77-84, January 2001.

[6] http://sequa.uib.cat/Avaluacio-docent/Avaluacio-docent-graus-imasters/ 